\title{
ARFI ULTRASOUND MONITORING OF HEMORRHAGE AND HEMOSTASIS IN VIVO IN CANINE VON WILLEBRAND DISEASE AND HEMOPHILIA
}

\author{
MALLORY R. SCOLA ${ }^{\star}$, TIMOTHY C. NICHOLS ${ }^{\dagger}, \ddagger$, HONGTU ZHU§, MELISSA C. CAUGHEY $\ddagger$, \\ ELIZABETH P. MERRICKS ${ }^{\dagger}$, ROBIN A. RAYMER ${ }^{\dagger}$, PARIS MARGARITIS ${ }^{\mathbb{1}}$, KATHERINE A.

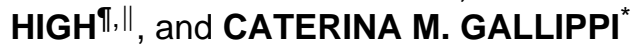 \\ *Joint Department of Biomedical Engineering, The University of North Carolina at Chapel Hill, \\ Chapel Hill, NC, USA \\ †Department of Pathology and Laboratory Medicine, The University of North Carolina at Chapel \\ Hill, Chapel Hill, NC, USA \\ ‡Department of Medicine, The University of North Carolina at Chapel Hill, Chapel Hill, NC, USA \\ $\S$ Department of Biostatistics, The University of North Carolina at Chapel Hill, Chapel Hill, NC, \\ USA \\ IDivision of Hematology, Children's Hospital of Philadelphia, PA, USA \\ "Howard Hughes Medical Institute, Children's Hospital of Philadelphia, PA, USA
}

\begin{abstract}
A validated method for assessing hemostasis in vivo is critical for testing the hemostatic efficacy of therapeutic agents designed for patients with bleeding disorders such as von Willebrand disease (VWD) and hemophilia A. We hypothesize that rate of bleeding and time to hemostasis can be monitored in vivo by acoustic radiation force impulse (ARFI) ultrasound. We performed ARFI imaging following 12-gauge needle puncture of hind limb muscle encompassing an $\sim 2 \mathrm{~mm}$ vein in six normal, eight naïve hemophilia A before and after infusing canine factor VIII, three hemophilia A expressing canine factor VIIa following gene transfer, and two naïve VWD dogs. Serial data sets were processed with custom software to (1) estimate the rate of hemorrhage and (2) estimate the time of hemostasis onset. The rate of hemorrhage during the first $30 \mathrm{~min}$ following puncture was markedly increased in the VWD dogs relative to normal but was not significantly different between normal, naïve hemophilia A or hemophilia A expressing cFVIIa. ARFI-derived times to hemostasis were significantly longer in naïve hemophilia A dogs than in normal dogs and were shortened by canine coagulation factors VIII and VIIa. These data support our hypothesis that rate of hemorrhage and time to hemostasis in vivo in response to a standardized hemostatic challenge can be detected by ARFI ultrasound in canine models of VWD and hemophilia. These data also suggest that the ARFI-monitored hemostatic challenge is relevant for in vivo testing of the hemostatic efficacy of therapeutic clotting factor replacement products used to treat inherited bleeding disorders.
\end{abstract}

(C) 2011 World Federation for Ultrasound in Medicine \& Biology

Address correspondence to: Caterina Gallippi, MD, Joint Department of Biomedical Engineering, The University of North Carolina at Chapel Hill and North Carolina State University, 152 MacNider Hall, CB 7575, Chapel Hill, NC 27599-7575 USA.

cmgallip@bme.unc.edu. 


\section{Keywords}

Acoustic radiation force impulse (ARFI) ultrasound imaging; Hemophilia; von Willebrand disease; Canine model; Soft-tissue bleeding; Hemostatic challenge; Replacement therapy; Gene therapy

\section{INTRODUCTION}

Hemophilia A and von Willebrand disease (VWD) are the most prevalent inherited bleeding disorders in humans. Hemophilia A occurs in approximately one in 5000 live male births and VWD in up to $1 \%$ in some populations (Rodeghiero et al. 1987; Mannucci and Tuddenham 2001; Federici 2007). Typically, patients with severe hemophilia suffer from spontaneous bleeding into joints, muscles and internal organs. Patients with VWD experience easy bruising and excessive bleeding from minor cuts and scratches, as well as bleeding from the mucous membranes of the nose, mouth and gastrointestinal tract (Kroll et al. 2001). Despite marked improvements in currently available (clotting factor replacement products [therapeutic clotting factor preparations that supply the deficient or anti-hemophilic protein to treat or prevent bleeding]), these inherited bleeding disorders are life-long and result in impaired quality of life, increased morbidity and mortality rates, extraordinary health care costs, significant health disparities and continued threat of exposure to infectious agents.

Documenting the hemostatic efficacy of new interventions to treat bleeding in hemophilia and VWD is hobbled by the lack of a validated method for assessing hemostasis in vivo. In general, hemophilia and VWD dog models, which closely resemble the respective human disorders, have been an outstanding preclinical predictor of the safety and efficacy of new treatments (Denis and Wagner 1999; Rawle and Lillicrap 2004; Nichols et al. 2009).

Historically, to document hemostatic efficacy in vivo, the primary saline bleeding time has been used in VWD (Nichols et al. 2011) and the secondary cuticle bleeding time has been used in hemophilia (Brinkhous et al. 1985, 2002). Both tests are difficult to reproduce in a given dog and vary greatly among dogs (Brinkhous et al. 1985, 1989, 2002). The lack of a validated, easily reproducible in vivo test of hemostasis is one of the major reasons that $e x$ vivo plasma based assays are accepted as a surrogate test of in vivo hemostasis. In fact, these plasma assays only detect the presence of clotting factor activity and do not measure hemostasis in vivo.

In this article, hemorrhage and bleeding are used interchangeably and are defined as the escape of blood from a vessel. Bleeding into muscles either spontaneously or following trauma to small veins and capillaries occurs in hemophilia (Ahlberg 1965; Bolton-Maggs and Pasi 2003) and severe type 3 VWD (Sadler et al. 2006; Federici 2007). Hemostasis is defined as the cessation of hemorrhage consisting of a primary phase, which includes a vasoconstrictive component and von Willebrand factor (VWF)-mediated platelet adhesion to sites of vessel injury (Sadler 2005; Nichols et al. 2008; Sadler 2008) and a secondary phase, which involves the generation of cross-linked insoluble fibrin in a tissue factorinitiated thrombin-mediated process (Monroe et al. 2002; Mackman et al. 2007). VWD is a disorder of primary hemostasis characterized by impaired platelet adhesion and (manifested phenotypically by excessive bleeding) that starts at the time of an injury. Hemophilia is a disorder of secondary hemostasis characterized by impaired thrombin generation and (manifested phenotypically by excessive bleeding) that is often delayed for variable amounts of time following an injury. 
We have previously demonstrated acoustic radiation force impulse (ARFI) ultrasound, an ultrasonic method for noninvasively interrogating the mechanical properties of tissue, for distinguishing subcutaneous and periarterial hemorrhage at femoral artery punctures in patients undergoing cardiac catheterization (Behler et al. 2009a, 2009b). We have now progressed to applying ARFI to monitoring rate of bleeding and detecting hemostasis following a standardized hemostatic challenge in relevant canine models of hemophilia A and VWD. Our data support ARFI's relevance to monitoring hemorrhage and hemostasis in vivo especially in preclinical testing of therapeutic agents.

\section{METHODS}

All dogs were treated according to the standards set in the Guide for Care and Use of Laboratory Animals (National Institutes of Health publication No. 85-23). All procedures and protocols were in accordance with institutional guidelines and approved by the Institutional Animal Care and Use Committee. All dogs were produced and maintained at the Francis Owen Blood Research Laboratory at the University of North Carolina at Chapel Hill. Dogs entered into this study were fully anesthetized with isoflurane to effect (usually $\sim 2 \%$ ) with constant monitoring of blood pressure, oxygen saturation and core body temperature. The dogs were placed in a prone position and the hind limb was shaved and prepped with an aseptic method, then, tethered to the table to prevent motion during imaging. The ultrasound transducer, held in place with a stereotactic clamp, was positioned above a peripheral vein ( $2 \mathrm{~mm}$ diameter) in the hind limb. A standardized hemostatic challenge was performed as follows: with ultrasound image guidance, a 12-gauge needle was used to puncture the hind limb muscle and vein five repeated times (Fig. 1). ARFI data and a spatially matched B-mode frame were serially acquired immediately before puncture, every minute for the first $15 \mathrm{~min}$ following puncture, every $2 \mathrm{~min}$ from 15 to $25 \mathrm{~min}$, then every $5 \mathrm{~min}$ thereafter for a total imaging period of $100 \mathrm{~min}$.

This hemostatic challenge coupled with ARFI imaging (to detect the rate of bleeding and time to hemostasis in the response to the hemostatic challenge) has been performed in the following series of dogs: normal $(n=6)$, naïve hemophilia A monitored for $30 \mathrm{~min}$ of bleeding then infused with canine FVIII to $11.3 \pm 1.8 \%$ activity (Chromogenix, $n=8$ ) to prevent formation of large hematomas, hemophilia A expressing canine factor FVIIa at 1.3$2 \mu \mathrm{g} / \mathrm{mL}$ (cFVIIa, $n=3$ ) (Margaritis et al. 2009) and naïve VWD $(n=2)$. The three hemophilia A dogs expressing cFVIIa underwent the full protocol twice, with imaging events 2 to 3 months apart.

ARFI imaging was performed using a Siemens SONOLINE Antares ${ }^{\mathrm{TM}}$ imaging system specially equipped for research purposes (Siemens Medical Solutions USA, Inc., Ultrasound Division) and a VF7-3 linear array transducer. The ARFI beam sequence consisted of two conventional two-cycle reference A-lines, followed by a 300-cycle (70 $\mu$ s) ARFI excitation impulse centered at $4.21 \mathrm{MHz}$, with an F/1.5 focal configuration. The excitation was followed by an ensemble of $60(6 \mathrm{~ms})$ conventional A-lines at a center frequency of 6.15 $\mathrm{MHz}$ and prf of $11 \mathrm{kHz}$. This ensemble was repeated in 40 locations spaced $0.53 \mathrm{~mm}$ apart laterally for a $2.1 \mathrm{~cm}$ lateral field of view (FOV). The focal depth was positioned on the near venous wall, typically $22 \mathrm{~mm}$ in depth. A $2 \mathrm{~cm}$ standoff pad was used as needed to optimize imaging.

Acquired RF data was transferred to a computational workstation for processing and analysis. One-dimensional (1-D) axial displacements were estimated along each acquired ensemble using cross-correlation with a $1.5 \lambda$ kernel, an interpolation factor of 4 and a search window length of $80 \mu \mathrm{m}$ (Pinton et al. 2006). Physiologic motion was rejected using a linear filter applied to the measured ARFI displacement profiles (Behler et al. 2007). 
Signal from hemorrhage was isolated using custom software (Scola et al. 2009). Our algorithm exploited measured 1-D axial displacements through time of ensemble acquisition and a priori information regarding soft tissue, hemorrhage and luminal blood displacement characteristics. Soft tissue was expected to have recovered by $4 \mathrm{~ms}$ after ARFI excitation, whereas hemorrhage was not expected to have recovered by $4 \mathrm{~ms}$ and luminal blood was expected to be flowing uninterrupted. Thus, it was anticipated that axial displacements measured $4 \mathrm{~ms}$ after ARFI excitation in the soft tissue would have low variance relative to hemorrhage. Similarly, the displacement variance of hemorrhage was anticipated to be low relative to luminal blood. These displacement characteristics are illustrated in Figure 1, which shows plots of displacement versus time (panel b) and second derivative of displacement vs. time (panel c) for representative hemorrhage (top), muscle and soft tissue (middle) and luminal blood (bottom) pixels. The second time derivative, a high-pass filtering operation (Bovik 2005), of hemorrhage has high variance relative to that of the soft tissue but low variance relative to that of luminal blood. An empirically derived upper threshold (on the variance of the second time derivative of displacement) was applied to exclude luminal blood, and an empirically derived lower threshold was applied to exclude soft tissue. The remaining pixels, identified as hemorrhage, were colored red on the matched B-mode images to create an ARFI/B-Mode hybrid image (Fig. 2).

Hemorrhage area was estimated by the number of identified hemorrhage pixels multiplied by $0.01 \mathrm{~mm}^{2}$, the spatial area spanned by each pixel. Plots of hemorrhage area vs. time were divided into 10-min intervals and fit using a linear-least squares approximation. The slope of each line segment was recorded as the hemorrhage rate of that 10-min interval and the time to $75 \%$ maximum hemorrhage area was defined as an approximation of time to hemostasis onset, as described by Figure 2 .

\section{Statistical analysis}

Statistical analyses were performed in R (R Foundation for statistical computing, Vienna). The two-sample $t$-test was the primary tool for comparing the differences of mean bleeding rate and time to hemostasis among the four groups. However, the two-sample Wilcoxon test with continuity correction was used to compare the differences of time to hemostasis between the normal group and the naïve hemophilia A group. Statistical significance was assumed for $p$ values below 0.05 .

\section{RESULTS}

ARFI-derived bleeding rates calculated over the first 30 min following puncture, depicted in Figure 3, were markedly increased in the VWD dogs relative to normal by a factor of $\sim 3(n=$ $2, p=0.0009$ ) (Table 1). Bleeding rates were not significantly different between normal dogs, hemophilia A dogs expressing cFVIIa at $1.3-2 \mu \mathrm{g} / \mathrm{mL}$ and naïve hemophilia A dogs before or after infusing cFVIII. The early data from these two VWD dogs suggest that the ARFI-derived bleeding rate will be applicable for detecting abnormal rates of bleeding in disorders of primary hemostasis and potentially for testing the hemostatic efficacy in vivo of relevant therapeutic agents.

ARFI-derived times to hemostasis, depicted in Figure 4, were significantly different between normal and naïve hemophilia A dogs (Table $1, p<0.00078$ ). Administration of FVIII to $11.3 \pm 1.8 \%$ of normal to treat the induced bleeding in these eight hemophilia A dogs shortened the time to hemostasis but to a range that was still significantly different than normal dogs $(p<0.036)$. Notably, expression of FVIIa at $1.3-2 \mu \mathrm{g} / \mathrm{mL}$ significantly shortened the time to hemostasis relative to naïve hemophilia A $(p<0.027)$, though the value was significantly different than normal dogs $(p<0.025)$. The dogs expressing FVIIa required no additional treatment to support hemostasis, a profound improvement in bleeding 
phenotype. Our time to hemostasis data points from two VWD dogs do not allow us to detect a relationship relative to normal dogs. These data support using the ARFI-derived time to hemostasis as an endpoint for detecting disorders of secondary hemostasis and for testing the hemostatic efficacy in vivo of relevant therapeutic agents.

Mean systolic and diastolic blood pressures during the imaging period for the five dog groups are reported in Table 1. Systolic pressures were not statistically significantly $(p>$ 0.05 ) different across dogs and neither were diastolic pressures. These data suggest that the impact of blood pressure on rate of hemorrhage and time to hemostasis was minimized by maintaining comparable systolic (108.05-154.11 mm Hg) and comparable diastolic (57.81$103.41 \mathrm{~mm} \mathrm{Hg}$ ) blood pressures for all dogs during the hemostatic challenge and ARFI monitoring period. Oxygen saturation was maintained above $90 \%$ and core body temperature was maintained between 100 and $102^{\circ} \mathrm{C}$ (normal range for dogs).

\section{DISCUSSION}

We hypothesized that rate of bleeding and time to hemostasis could be monitored in vivo by ARFI ultrasound. We have detected significant differences in the rate of bleeding between normal and VWD dogs. Also, significantly longer times to hemostasis were detected in naïve hemophilia A dogs relative to normal. These data support our hypothesis and provide justification for validating the ARFI monitored hemostatic challenge as an in vivo test of hemostasis. Because no comparable in vivo test for hemostasis is currently available, we were unable to compare our measurements with published values for time to hemostasis and rate of hemorrhage.

Von Willebrand factor mediates platelet adhesion at sites of endothelial disruption, especially under high shear conditions (Sadler 2005, 2008; Nichols et al. 2008). The primary hemostatic defect that is phenotypically characteristic of VWD is most likely due to inadequate formation of a platelet plug at sites of vascular perforation. Our results have shown markedly increased rates of bleeding with the ARFI monitored hemostatic challenge when VWD dogs are compared with normal or hemophilia A. Although the finding of increased rates of bleeding in VWD will need to be confirmed in additional VWD dogs, these preliminary observations support the hypothesis that increased rate of bleeding with this hemostatic challenge is due to a defect in primary hemostasis resulting from the absence of VWF. Confirmation of this hypothesis will require determining if normalization of the defect in primary hemostasis can be accomplished by replacement of VWF function in these VWD dogs.

In contrast to VWD, hemophilic subjects have normal VWF levels and, therefore, normal VWF-mediated platelet plug formation but have a failure of secondary hemostasis (Kazazian et al. 2001; Bolton-Maggs and Pasi 2003). Impaired or absent coagulation factor VIII in hemophilia A results in inadequate amounts of thrombin formation and, consequently, defective stabilization of the platelet plug by fibrin. Our results have shown significantly longer time to hemostasis in hemophilia A with the ARFI monitored hemostatic challenge when naïve hemophilia A dogs are compared with normal. Most importantly, infusion of cFVIII to $11.3 \pm 1.8 \%$ of normal activity markedly shortened the time to hemostasis and expression of cFVIIa at therapeutic levels (Brinkhous et al. 1989; Margaritis et al. 2009) shortened the time to hemostasis to the range found in normal dogs. Further studies are planned to determine if infusion of higher levels of cFVIII in hemophilia A dogs would support a time to hemostasis more like that in normal dogs. Also of note, the hemophilia A dogs expressing cFVIIa required no additional therapeutic agents (i.e., $\mathrm{cFVIII)}$ to support hemostasis. This result suggests that expression of cFVIIa by gene therapy supports hemostasis in vivo and corroborate the correction of the hemophilic phenotype observed in 
these dogs with the partial correction of the whole blood clotting time and absence of spontaneous bleeding events (Margaritis et al. 2009). Bleeding in naïve hemophilia A dogs was limited to a 30-min observation period, after which time the cFVIII was administered to the dogs to prevent the formation of a large hematoma. This approach did not allow us to measure times to hemostasis beyond $30 \mathrm{~min}$ in the naïve hemophilia A dogs. Notably, none of the eight naïve hemophilia A dogs had achieved hemostasis by 30 min according to our ARFI definition. These findings support the hypothesis that in naïve hemophilia $\mathrm{A}$, the time to hemostasis with this hemostatic challenge is due to a defect in secondary hemostasis due to the absence of FVIII and impaired thrombin formation. The corollary is that support of thrombin formation by infusion of cFVIII or FVIIa improved secondary hemostasis reflected in the reduced ARFI-derived times to hemostasis.

As with any radiation-force-based application, it is important to be mindful of potential thermal or mechanical bioeffects. Heating associated with our applied ARFI excitation impulses is estimated to be $0.06^{\circ} \mathrm{C}$ in human tissue, neglecting perfusion and other cooling effects (Palmeri et al. 2004; Palmeri and Nightingale 2004). In addition, we measured mechanical displacements of less than $10 \mu \mathrm{m}$ in areas of blood. We, therefore, do not expect that clotting times were affected by either heating or by mechanical disturbances induced by ARFI.

One source of error in our results is a limited two-dimensional (2-D) field of view (FOV). It is apparent in Figure 2 that hemorrhage areas extend beyond the white vertical lines, which demarcate the ARFI lateral range. Hemorrhage outside the ARFI lateral range would result in smaller detected hemorrhage areas and may distort time to hemostasis and hemorrhage rate estimates. We will consider broadening the ARFI lateral range in future studies. Despite this error, our time to hemostasis and hemorrhage rate estimates follow the trends expected for established hemophilia A and VWD phenotypes.

An area for enhanced performance of in vivo ARFI hemostasis assessment is threedimensional (3-D) imaging. Three-dimensional imaging could improve estimation of time to hemostasis and hemorrhage rate by monitoring hemorrhage volume as opposed to crosssectional area. Hemorrhage volume is likely to be a more accurate indication of active bleeding given the asymmetric geometries of hematomas. Three-dimensional ARFI imaging would also support quantification of hemorrhage volume, a clinically meaningful metric (Gutierrez et al. 2004).

\section{CONCLUSIONS}

This preclinical pilot feasibility study in relevant canine models of inherited bleeding disorders supports that ARFI ultrasound is a viable imaging method for in vivo detection of rate of hemorrhage and onset of hemostasis following a standardized hemostatic challenge. These results are consistent with our previous pilot clinical demonstration of ARFI imaging for evaluating hemostasis at femoral artery puncture, which showed shortened ARFI-derived times to hemostasis with application of a hemostatic dressing relative to standard of care manual compression alone (Behler et al. 2009b). This work strongly supports ARFI's relevance for assessing the hemostatic efficacy in vivo of therapies for inherited bleeding disorders. A rational extension of this work would be to apply ARFI monitoring of hemorrhage in acquired bleeding disorders such as with antiplatelet and anticoagulant therapies, massive trauma or surgery. 


\section{Acknowledgments}

The authors thank Siemens Medical Solutions USA, Inc. Ultrasound Division for their in kind support. This work was supported by Novo Nordisk (Gallippi), NIH/NHLBI HL063098 (Nichols), NIH/NHLBI P01 HL074124 (High); and the NIH Integrated Biomedical Research Training Program (IBRTP) (Marzluff).

\section{REFERENCES}

1. Ahlberg A, Haemophilia in Sweden. VII. Incidence, treatment and prophylaxis of arthropathy and other musculo-skeletal manifestations of haemophilia A and B. Acta Orthop Scand Suppl. 1965; 77(Suppl):3-132. [PubMed: 5854304]

2. Behler RH, Merriciks EP, Nichols TC, Gallippi CM. A rigid wall approach to physiological motion rejection in arterial ARFI imaging: Simulation and in vivo demonstration. Int IEEE Ultrason Symp Proc. 2007:359-364.

3. Behler RH, Scola MR, Nichols TC, Bellinger DA, Gallippi CM. ARFI ultrasound for in vivo hemostasis assessment postcardiac catheterization, part I: Preclinical studies. Ultrason Imaging. 2009a; 31:153-158. [PubMed: 19771958]

4. Behler RH, Scola MR, Nichols TC, Caughey MC, Fisher MW, Zhu H, Gallippi CM. ARFI ultrasound for in vivo hemostasis assessment postcardiac catheterization, part II: Pilot clinical results. Ultrason Imaging. 2009b; 31:159-171. [PubMed: 19771959]

5. Bolton-Maggs PH, Pasi KJ. Haemophilias A and B. Lancet. 2003; 361:1801-1809. [PubMed: 12781551]

6. Bovik, AC. Elsevier Academic Press; Amsterdam; Boston, MA: 2005. Handbook of image and video processing.

7. Brinkhous K, Sandberg H, Widlund L, Read M, Nichols T, Sigman J, Oswaldsson U, Schaub RG, Mikaelsson M. Preclinical pharmacology of albumin-free B-domain deleted recombinant factor VIII. Semin Thromb Hemost. 2002; 28:269-272. [PubMed: 12098087]

8. Brinkhous KM, Hedner U, Garris JB, Diness V, Read MS. Effect of recombinant factor VIIa on the hemostatic defect in dogs with hemophilia A, hemophilia B, and von Willebrand disease. Proc Natl Acad Sci U S A. 1989; 86:1382-1386. [PubMed: 2784006]

9. Brinkhous KM, Sandberg H, Garris JB, Mattsson C, Palm M, Griggs T, Read MS. Purified human factor VIII procoagulant protein: Comparative hemostatic response after infusions into hemophilic and von Willebrand disease dogs. Proc Natl Acad Sci U S A. 1985; 82:8752-8756. [PubMed: 3936044]

10. Denis CV, Wagner DD. Insights from von Willebrand disease animal models. Cell Mol Life Sci. 1999; 56:977-990. [PubMed: 11212329]

11. Federici, AB. Textbook of Hemophilia. Blackwell Publishing Ltd; 2007. Classification and clinical aspects of Von Willebrand Disease; p. 279-284.

12. Gutierrez G, Reines HD, Wulf-Gutierrez ME. Clinical review: Hemorrhagic shock. Crit Care. 2004; 8:373-381. [PubMed: 15469601]

13. Kazazian, HH.; Tuddenham, EGD.; Antonarakis, SE. Hemophilia A: Deficiency of coagulation factor VIII. McGraw-Hill; 2001.

14. Kroll, MH.; Palmer, JG.; Herion, JC. Manual of coagulation disorders. Blackwell Science; Malden, MA: 2001.

15. Mackman N, Tilley RE, Key NS. Role of the extrinsic pathway of blood coagulation in hemostasis and thrombosis. Arterioscler Thromb Vasc Biol. 2007; 27:1687-1693. [PubMed: 17556654]

16. Mannucci PM, Tuddenham EG. The hemophilias-From royal genes to gene therapy. N Engl J Med. 2001; 344:1773-1779. [PubMed: 11396445]

17. Margaritis P, Roy E, Aljamali MN, Downey HD, Giger U, Zhou S, Merricks E, Dillow A, Ezban M, Nichols TC, High KA. Successful treatment of canine hemophilia by continuous expression of canine FVIIa. Blood. 2009; 113:3682-3689. [PubMed: 19109232]

18. Monroe DM, Hoffman M, Roberts HR. Platelets and thrombin generation. Arterioscler Thromb Vasc Biol. 2002; 22:1381-1389. [PubMed: 12231555] 
19. Nichols T, Bellinger D, Merricks E, Raymer R, Kloos M, DeFriess N, Ragni M, Griggs T. Porcine and canine von Willebrand factor and von Willebrand disease: Hemostasis, thrombosis, and atherosclerosis studies. Thrombosis. 2011;2010:11.

20. Nichols TC, Dillow AM, Franck HW, Merricks EP, Raymer RA, Bellinger DA, Arruda VR, High KA. Protein replacement therapy and gene transfer in canine models of hemophilia A, hemophilia B, von Willebrand disease, and factor VII deficiency. Ilar J. 2009; 50:144-167. [PubMed: 19293459]

21. Nichols WL, Hultin MB, James AH, Manco-Johnson MJ, Montgomery RR, Ortel TL, Rick ME, Sadler JE, Weinstein M, Yawn BP. von Willebrand disease (VWD): Evidence-based diagnosis and management guidelines, the National Heart, Lung, and Blood Institute (NHLBI) Expert Panel report (USA). Haemophilia. 2008; 14:171-232. [PubMed: 18315614]

22. Palmeri ML, Frinkley KD, Nightingale KR. Experimental studies of the thermal effects associated with radiation force imaging of soft tissue. Ultrason Imaging. 2004; 26:100-114. [PubMed: 15344414]

23. Palmeri ML, Nightingale KR. On the thermal effects associated with radiation force imaging of soft tissue. IEEE Trans Ultrason Ferroelectr Freq Control. 2004; 51:551-565. [PubMed: 15217233]

24. Pinton GF, Dahl JJ, Trahey GE. Rapid tracking of small displacements with ultrasound. IEEE Trans Ultrason Ferroelectr Freq Control. 2006; 53:1103-1117. [PubMed: 16846143]

25. Rawle FE, Lillicrap D. Preclinical animal models for hemophilia gene therapy: Predictive value and limitations. Semin Thromb Hemost. 2004; 30:205-213. [PubMed: 15118932]

26. Rodeghiero F, Castaman G, Dini E. Epidemiological investigation of the prevalence of von Willebrand's disease. Blood. 1987; 69:454-459. [PubMed: 3492222]

27. Sadler JE. von Willebrand factor: Two sides of a coin. J Thromb Haemost. 2005; 3:1702-1709. [PubMed: 16102036]

28. Sadler JE. Von Willebrand factor, ADAMTS13, and thrombotic thrombocytopenic purpura. Blood. 2008; 112:11-18. [PubMed: 18574040]

29. Sadler JE, Budde U, Eikenboom JC, Favaloro EJ, Hill FG, Holmberg L, Ingerslev J, Lee CA, Lillicrap D, Mannucci PM, Mazurier C, Meyer D, Nichols WL, Nishino M, Peake IR, Rodeghiero F, Schneppenheim R, Ruggeri ZM, Srivastava A, Montgomery RR, Federici AB. Update on the pathophysiology and classification of von Willebrand disease: A report of the Subcommittee on von Willebrand Factor. J Thromb Haemost. 2006; 4:2103-2114. [PubMed: 16889557]

30. Scola, MR.; Mauldin, E.; Gallippi, CM. Proceedings of 2009 IEEE International Ultrasonics Symposium, 20-23 Sept. 2009. IEEE Ultrason Symp; Rome, Italy: 2009. Analysis of crosscorrelation coefficients for subcutaneous blood signal detection by ARFI Imaging; p. 1883-1886.

Ultrasound Med Biol. Author manuscript; available in PMC 2014 February 18. 


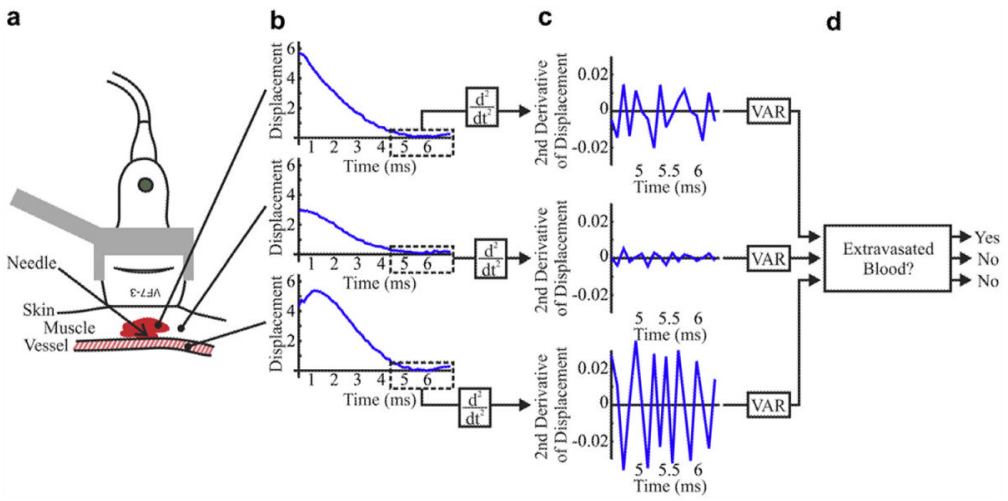

Fig 1.

Methods of blood signal detection in acoustic radiation force impulse (ARFI) ultrasound. (a) The imaging transducer is positioned above punctured muscle and surrounding soft tissue and small ( $2 \mathrm{~mm})$ vein. (b) ARFI displacement profiles arising from regions of hemorrhage and tissue (top), muscle and soft tissue alone (middle) and luminal blood (bottom) exhibit late displacement variations (boxed) that are highlighted by (c) the second time derivative. (d) Applying upper and lower thresholds to the variance of the second time derivative of displacement distinguishes hemorrhage from soft tissue and luminal blood. 


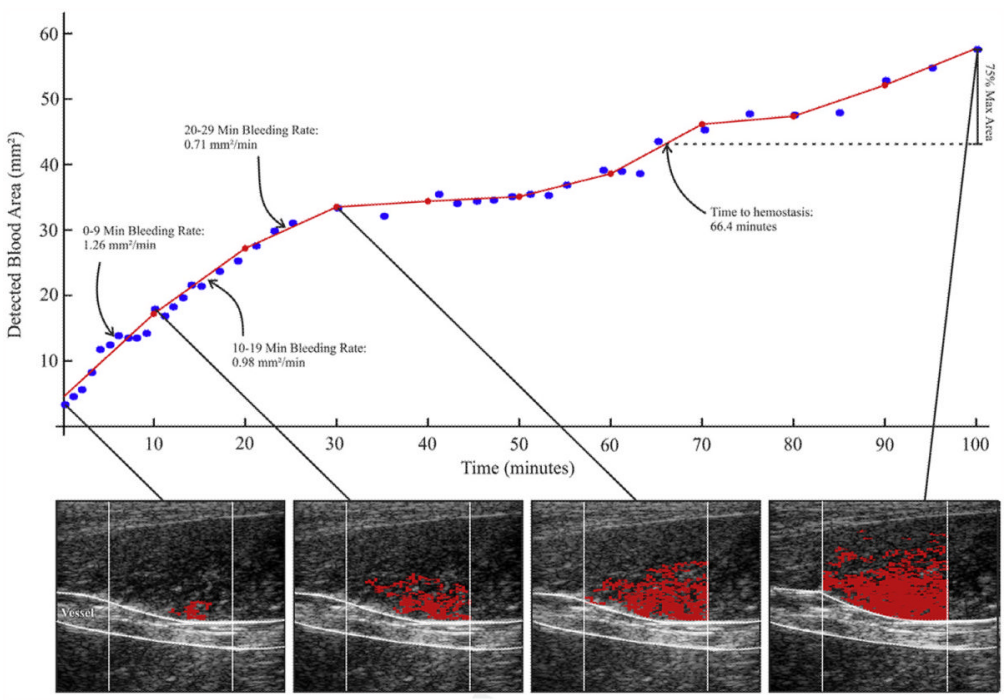

Fig 2.

Methods of acoustic radiation force impulse (ARFI)hemorrhage rate and time to hemostasis estimation. (Bottom row) In serially acquired ARFI frames in a naïve hemophilia A dog, hemorrhage pixels (red) are identified (Fig. 1) and associated cross-sectional area (CSA) calculated. (Top graph) CSA is plotted versus time of image acquisition (blue dots). The CSA plot is subdivided into 10 min intervals and a line is fit using a linear least-squares regression approximation (red lines). The slopes of the lines are recorded as the hemorrhage rate $\left(\mathrm{mm}^{2} / \mathrm{min}\right)$. Time to hemostasis onset is estimated as time to $75 \%$ of the maximum observed hemorrhage CSA. Please note, the dog was infused with cFVIII to $11.3 \pm 1.8 \%$, at $30 \mathrm{~min}$. 


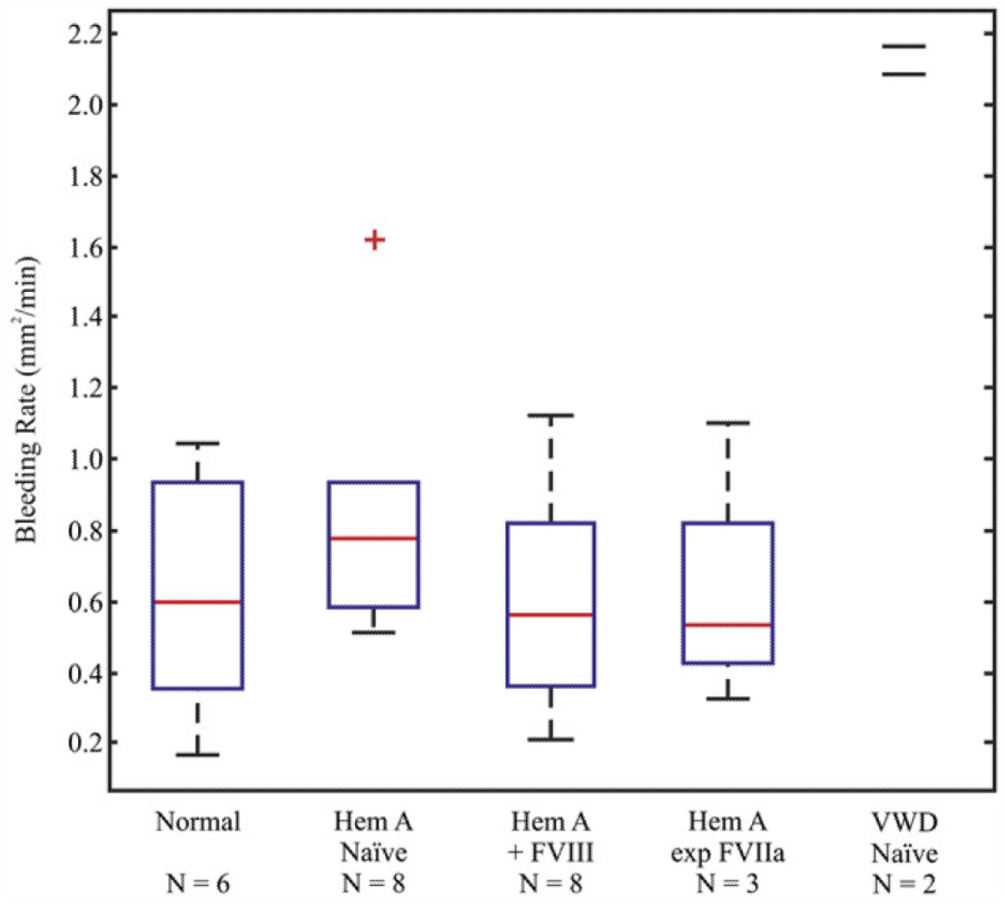

Fig 3.

Acoustic radiation force impulse (ARFI)-derived hemorrhage rate. Box-plot displaying the median (red-line), 25th and 75th percentiles (blue box), range of observed values (dashed black lines) and outliers (red "plus") for the hemorrhage rate in the first 30 min following puncture for all normal, naïve hemophilia A, naïve hemophilia A treated with FVIII to 11.3 $\pm 1.8 \%$, cFVIIa expressing hemophilia A and naïve Willebrand disease (VWD) dogs. 


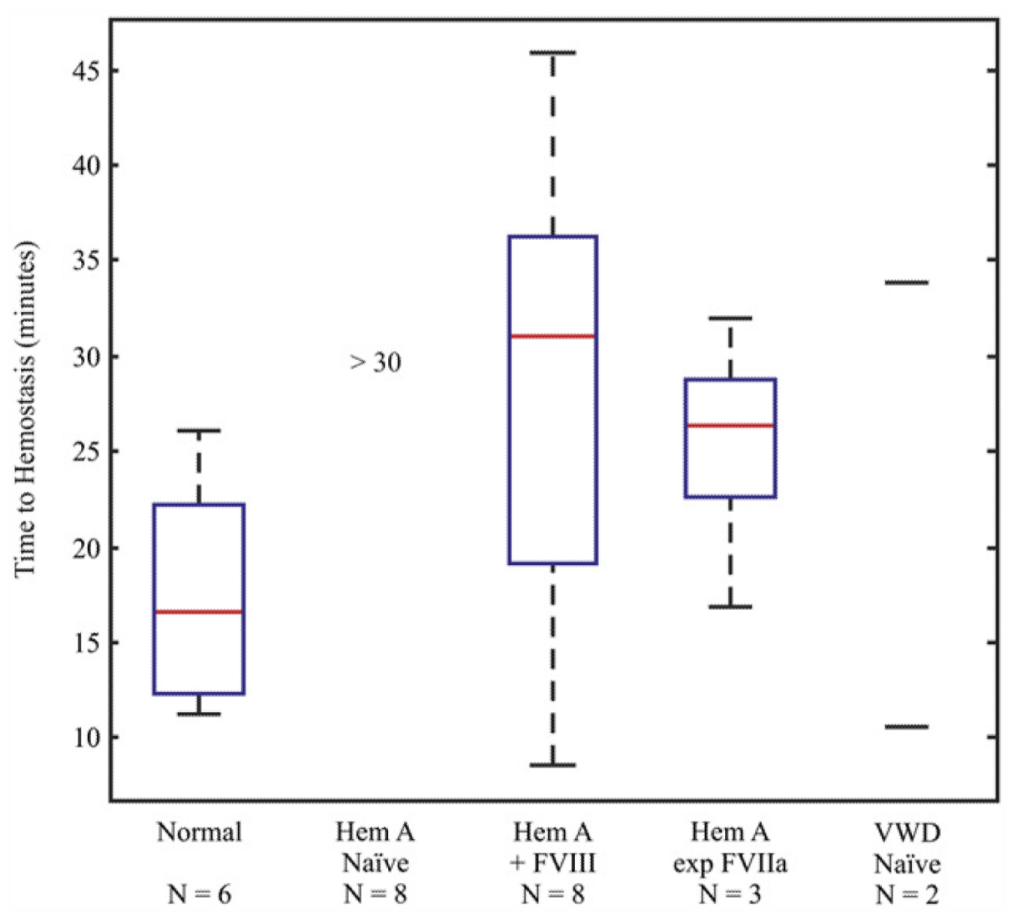

Fig 4.

Acoustic radiation force impulse (ARFI)-derived time to hemostasis. Box-plot displaying the median (red-line), 25th and 75th percentiles (blue box) and range of observed values (dashed black lines) for the time to hemostasis for all normal, naïve hemophilia A, naïve hemophilia A treated with FVIII to $11.3 \pm 1.8 \%$, cFVIIa expressing hemophilia A and naïve Willebrand disease (VWD) dogs. 


\section{Table 1}

Bleeding rates and time to hemostasis from ARFI-monitored hemostatic challenge by dog phenotype (mean \pm standard deviation)

\begin{tabular}{|c|c|c|c|c|}
\hline Dog phenotype & $\mathbf{n}$ & $\begin{array}{l}\text { Mean bleeding rate }\left(\mathrm{mm}^{2} /\right. \\
\text { min) }\end{array}$ & $\begin{array}{l}\text { Time to hemostasis } \\
(\text { (min) })^{*}\end{array}$ & Mean BP $(\mathbf{m m} \mathbf{H g})$ \\
\hline 1. Normal & 6 & $0.6174 \pm 0.33254^{*}$ & $17.52 \pm 6.05^{\S}$ & $134.38 / 82.69 \pm 18.41 / 21.16^{q I}$ \\
\hline 2. Naïve hemophilia A & 8 & $0.86887 \pm 0.39963^{\dagger}$ & $>30^{\S}$ & $128.20 / 77.37 \pm 10.59 / 16.89 \pi$ \\
\hline $\begin{array}{l}\text { 3. Naïve hemophilia A treated with FVIII } \\
\text { to } 11.3 \pm 1.8 \%\end{array}$ & 8 & $0.60819 \pm 0.32415^{\ddagger}$ & $28.40 \pm 12.39 \S$ & $135.98 / 88.27 \pm 8.24 / 13.28 \pi$ \\
\hline $\begin{array}{l}\text { 4. Hemophilia A expressing FVIIa } \\
\text { (Margaritis et al. 2009) at } 1.3-2 \mu \mathrm{g} / \mathrm{mL}\end{array}$ & $3^{\dagger}$ & $0.62228 \pm 0.33465^{\ddagger}$ & $25.50 \pm 5.60^{\S}$ & $127.53 / 77.33 \pm 11.51 / 4.38^{I I}$ \\
\hline 5. Naïve VWD & 2 & $2.0890,2.1625^{\star}$ & $10.60,33.80$ & $119.04 / 66.06,104.47 / 57.88^{q}$ \\
\hline
\end{tabular}

$\mathrm{ARFI}=$ acoustic radiation force impulse; $\mathrm{VWD}=\mathrm{Willebrand}$ disease

* ARFI time to hemostasis is defined as that required to bleed $75 \%$ of the total detected area of hemorrhage.

${ }^{\dagger}$ Mean from two separate procedures on each dog. No additional plasma therapy was required in these dogs.

${ }^{+}$Mean bleeding rate comparisons 1 vs. $2(p<0.196), 1$ vs. $3(p<0.952), 1$ vs. $4(p<0.970), 1$ vs. $5(p<0.0009)$.

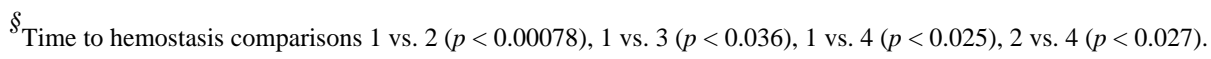

${ }^{I l}$ Blood pressure comparisons 1 vs. $2(p<0.508), 1$ vs. 3 ( $\left.p<0.704\right), 1$ vs. $4(p<0.445), 1$ vs. $5(p<0.391)$. 